\title{
BOUNDED POLYNOMIAL VECTOR FIELDS
}

\author{
ANNA CIMA AND JAUME LLIBRE
}

\begin{abstract}
We prove that, for generic bounded polynomial vector fields in $\mathbf{R}^{n}$ with isolated critical points, the sum of the indices at all their critical points is $(-1)^{n}$. We characterize the local phase portrait of the isolated critical points at infinity for any bounded polynomial vector field in $\mathbf{R}^{2}$. We apply this characterization to show that there are exactly seventeen different behaviours at infinity for bounded cubic polynomial vector fields in the plane.
\end{abstract}

\section{INTRODUCTION}

Let $X: U \rightarrow \mathbf{R}^{k}$ be a vector field where $U$ is an open set of $\mathbf{R}^{k}$. Let $\gamma(t)=\gamma(t, x)$ be the integral curve of $X$ such that $\gamma(0)=x$. Let $I_{x}$ be its maximal interval of definition. We shall say that $X$ is a bounded vector field if for all $x \in U$, there exists some compact set $K \subset U$ such that $\gamma(t) \in K$ for each $t \in I_{x} \cap(0,+\infty)$.

In $\S 1$ we introduce the stereographic compactification of $X, s(X)$. We then use the index formula of Bendixson and the Poincare-Hopf theorem to prove the following result:

Proposition A. Let $X$ be a bounded polynomial vector field in the plane. If all the critical points of $s(X)$ are isolated, then the sum of the indices at all those critical points is 1 .

In $\S 2$ we use the Poincare compactification of $X, p(X)$, to characterize the local phase portrait of the isolated critical points at infinity for bounded polynomial vector fields $X=(P, Q)$ in the plane. The degree $n$ of $X$ is defined by $n=\max \{$ degree $P$, degree $Q\}$. We denote by $i_{X}(q)$ the index of $X$ at a critical point $q$ of $X$. We then prove the following theorem:

Theorem B. Let $X$ be a bounded polynomial vector field in the plane. If $q$ is an isolated infinite critical point of $X$, then

(a) The local phase portrait of $p(X)$ at $q$ is described in Figure 2.2 (resp. Figure 2.4) when the degree of $X$ is even (resp. odd).

Received by the editors July 12,1988. The contents of this paper have been presented to the meeting "Qualitative Theory of Differential Equations" in Szeged, Hungary, August 1988.

1980 Mathematics Subject Classification (1985 Revision). Primary 34C05; Secondary 58F14.

Key words and phrases. Bounded vector field, index, blow-up.

The two authors have been partially supported by a CICYT grant PB 86-0351. This work is a chapter of the Ph.D. thesis of the first author; see [C]. 
(b) $i_{p(X)}(q)=0$ when the degree of $X$ is even.

(c) $i_{p(X)}(q)=i_{p(X) \mid \mathbf{S}^{\prime}}(q)$ when the degree of $X$ is odd. Here, $p(X) \mid \mathbf{S}^{1}$ denotes the restriction of the vector field $p(X)$ to the equator $\mathbf{S}^{1}$ of the Poincare sphere.

In $\S 3$ we use Theorem B to show that there are seventeen different behaviours at infinity for bounded polynomial vector fields of degree three. This statement can be formalized as

Theorem C. The phase-portrait in a neighbourhood of infinity for any bounded cubic system with isolated infinite critical points is topologically equivalent to

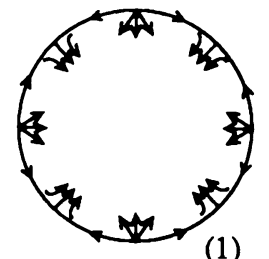

(1)

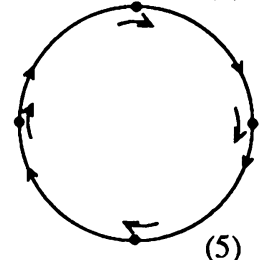

(5)
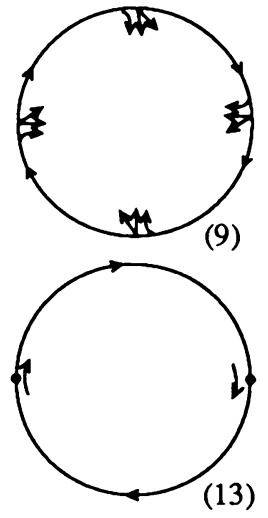

(13)

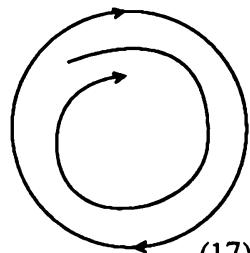

(17)

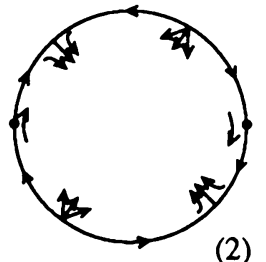

(2)

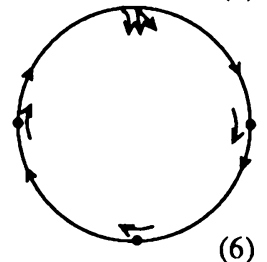

(6)

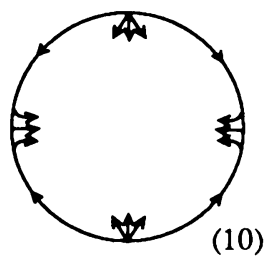

(10)

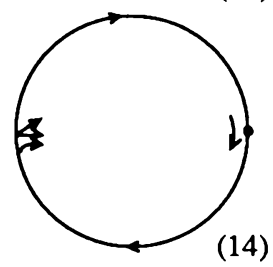

(14)

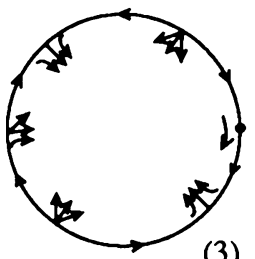

(3)

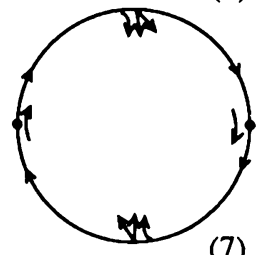

(7)

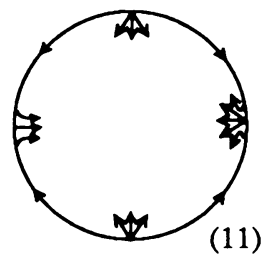

(11)

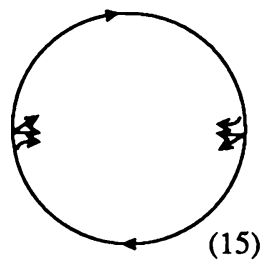

(15)

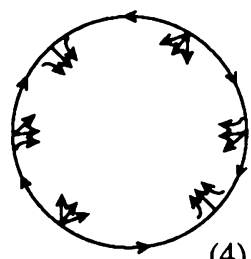

(4)

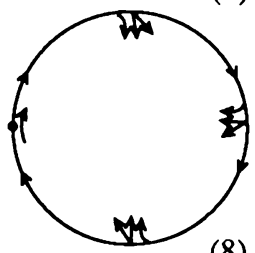

(8)

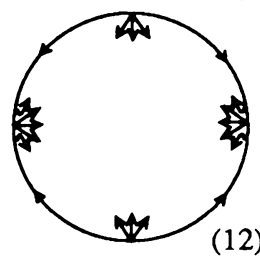

(12)

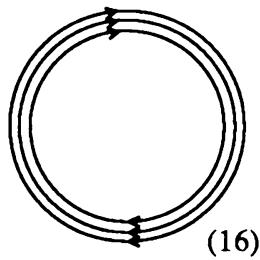

FIGURE 1.1. The phase portraits in a neighbourhood of infinity for the bounded cubic vector fields with isolated infinite critical points 
a particular picture in Figure 1.1. All these behaviours are realized by the systems given in Table 1.1.

TABLE 1.1. System $X_{i}$ realizes the behaviour (i) of Figure 1.1 for $i=1,2, \ldots, 17$.

$$
\begin{aligned}
& X_{1}:\left\{\begin{array}{l}
\dot{x}=-100 x^{3}+51 x^{2} y-24 y^{3}, \\
\dot{y}=24 x^{3}-100 x^{2} y-51 x y^{2} .
\end{array}\right. \\
& \begin{array}{l}
X_{2}:\left\{\begin{array}{l}
\dot{x}=-2 x y^{2}+y^{3}, \\
\dot{y}=x+x y^{2}-2 y^{3}
\end{array}\right. \\
X_{4}:\left\{\begin{array}{c}
\dot{x}=-x^{3}+(1 / 2) x^{2} y \\
-x y^{2}-y^{3}, \\
\dot{y}=-x^{2} y-(1 / 2) x y^{2}-y^{3} .
\end{array}\right.
\end{array} \\
& X_{3}:\left\{\begin{array}{l}
\dot{x}=x y+2 x^{2}-2 x y^{2}+y^{3}, \\
\dot{y}=x^{2}+x y^{2}-2 y^{3} .
\end{array}\right. \\
& \begin{array}{l}
X_{2}:\left\{\begin{array}{l}
\dot{x}=-2 x y^{2}+y^{3}, \\
\dot{y}=x+x y^{2}-2 y^{3}
\end{array}\right. \\
X_{4}:\left\{\begin{array}{c}
\dot{x}=-x^{3}+(1 / 2) x^{2} y \\
-x y^{2}-y^{3}, \\
\dot{y}=-x^{2} y-(1 / 2) x y^{2}-y^{3} .
\end{array}\right.
\end{array} \\
& X_{5}:\left\{\begin{array}{l}
\dot{x}=(-1 / 2) x^{2} y+3 / 2 x y^{2}-y^{3}, \\
\dot{y}=(1 / 2) x y^{2}-(1 / 2) y^{3}+x .
\end{array}\right. \\
& X_{6}:\left\{\begin{aligned}
\dot{x}= & -x+7 x^{2}-10 x y \\
& -y^{2}+x y^{2}-y^{3}, \\
\dot{y}= & (5 / 2) x+x^{2}+2 x y
\end{aligned}\right. \\
& X_{7}:\left\{\begin{array}{l}
\dot{x}=y+3 x^{2} y-x^{3}, \\
\dot{y}=-x^{2} y-3 x y^{2} .
\end{array}\right. \\
& X_{9}:\left\{\begin{array}{l}
\dot{x}=-x^{3}-6 x y^{2}+6 y^{3}, \\
\dot{y}=-x^{2} y-6 x y^{2}+6 y^{3} .
\end{array}\right. \\
& X_{11}:\left\{\begin{array}{l}
\dot{x}=-x-x y^{2}-4 y^{3}, \\
\dot{y}=-5 y^{3}+x y .
\end{array}\right. \\
& X_{13}:\left\{\begin{array}{l}
\dot{x}=3 x y^{2}+y^{3}, \\
\dot{y}=-3 x y^{2}-x .
\end{array}\right. \\
& X_{15}:\left\{\begin{array}{l}
\dot{x}=-x^{3}-y^{3}, \\
\dot{y}=-x^{2} y .
\end{array}\right. \\
& X_{17}:\left\{\begin{array}{l}
\dot{x}=-x^{3}-x^{2} y-y^{3}, \\
\dot{y}=x^{3}-x^{2} y+x y^{2} .
\end{array}\right. \\
& X_{8}:\left\{\begin{array}{c}
\quad-7 y^{2}+x y^{2}-y^{3} . \\
\dot{x}=4 x^{2}-x y-y^{3}, \\
\dot{y}=x^{2}+x y^{2}-2 y^{3} .
\end{array}\right. \\
& X_{10}:\left\{\begin{array}{l}
\dot{x}=-2 x^{3}+y^{3}, \\
\dot{y}=x^{3}-2 x^{2} y .
\end{array}\right. \\
& X_{12}:\left\{\begin{array}{l}
\dot{x}=-4 x+3 x y^{2}-4 y^{3}, \\
\dot{y}=-y-2 y^{2}-y^{3} .
\end{array}\right. \\
& X_{14}:\left\{\begin{array}{l}
\dot{x}=x^{2}-x y-x y^{2}-y^{3}, \\
\dot{y}=x^{2}+x y^{2}-y^{3} . \\
\dot{x}=y^{3} \\
\dot{y}=-x^{3} .
\end{array}\right.
\end{aligned}
$$

In $\S 4$ we prove that Proposition A also holds for a generic family of bounded polynomial vector fields in $\mathbf{R}^{n}$. We call $\mathscr{X}_{m}$ the set of all polynomial vector fields $X=\left(P^{1}, P^{2}, \ldots, P^{n}\right)$ defined on $\mathbf{R}^{n}$ with degree $P^{i}=m_{i}$ and $m=$ $\left(m_{1}, m_{2}, \ldots, m_{n}\right)$. We denote by $P_{m_{i}}^{i}$ the homogeneous part of degree $m_{i}$ of $P^{i}$. Let $\mathscr{G}_{m}$ be the set of all $X \in \mathscr{X}_{m}$ such that the system $P_{m_{i}}^{i}=0$ for $i=1,2, \ldots, n$ has only the trivial solution $x_{1}=x_{2}=\cdots=x_{n}=0$. In other words when $X \in \mathscr{G}_{m}$ all the solutions of system $P^{i}=0$, for $i=1,2, \ldots, n$, lie in the "finite part" of $\mathbf{R}^{n}$; for more details see Appendix 2. In the case where $m_{i}=m_{j}$ for all $i, j=1,2, \ldots, n$ and $X \in \mathscr{G}_{m}$, the linear part of $p(X)$, at the critical points $q$ at infinity, has an eigenvalue different from zero. It is precisely this eigenvalue that determines whether the orbits of $X$ go off or come from infinity in the direction determined by $q$ (see Lemma 4.1). It is 
proved (see Appendix 2) that $X \in \mathscr{G}_{m}$ is a generic condition. We then use the Poincaré compactification in $\mathbf{R}^{n}$ (see Appendix 1), the Poincaré-Hopf theorem and the Center Manifold Theorem (see [GH]) to prove the following theorem.

Theorem D. Let $X \in \mathscr{G}_{m}$ be a bounded polynomial vector field in $\mathbf{R}^{n}$ such that all the critical points of $p(X)$ are isolated and $\operatorname{degree}\left(P^{i}\right)=m$, for $i=$ $1,2, \ldots, n$. Then

$$
\sum_{f} i_{X}=(-1)^{n}
$$

where the sum is defined over the indices of all the critical points of $X$.

We note that Theorem $\mathrm{D}$ is a (nonvacuous) statement for odd degree polynomial vector fields (see Proposition 4.2).

\section{INDEX FOR BOUNDED POLYNOMIAL VECTOR FIELDS IN THE PLANE}

Let $X=(P, Q)$ be a polynomial vector field of degree $n$ in the plane and let $\mathbf{S}^{2}$ be the two-sphere on $\mathbf{R}^{3}$ defined by the set $\left\{y \in \mathbf{R}^{3}: y_{1}^{2}+y_{2}^{2}+\left(y_{3}-1 / 2\right)^{2}=\right.$ $1 / 4\}$. The plane $\mathbf{R}^{2}$ may be identified with the sphere $\mathbf{S}^{2}$ with the "north pole" $p=(0,0,1)$ removed. This identification is accomplished by means of the stereographic projection which assigns to each point $\left(x_{1}, x_{2}\right) \in \mathbf{R}^{2}$ the point $\left(y_{1}, y_{2}, y_{3}\right) \in \mathbf{S}^{2}$ through the relations $x_{1}=y_{1} /\left(1-y_{3}\right), x_{2}=y_{2} /\left(1-y_{3}\right)$. Let $\widetilde{X}=\left(F_{1}, F_{2}, F_{3}\right)$ be the induced vector field on $\mathbf{S}^{2} \backslash\{p\}$. It can be shown that the components $F_{i}\left(y_{1}, y_{2}, y_{3}\right)=d y_{i} / d t$, for $i=1,2,3$, are

$$
\begin{gathered}
F_{1}\left(y_{1}, y_{2}, y_{3}\right)=\left(1-y_{3}-y_{1}^{2}\right), \quad P\left(\frac{y_{1}}{1-y_{3}}, \frac{y_{2}}{1-y_{3}}\right)-y_{1} y_{2}, \\
Q\left(\frac{y_{1}}{1-y_{3}}, \frac{y_{2}}{1-y_{3}}\right), \\
F_{2}\left(y_{1}, y_{2}, y_{3}\right)=-y_{1} y_{2}, \quad P\left(\frac{y_{1}}{1-y_{3}}, \frac{y_{2}}{1-y_{3}}\right)+\left(1-y_{3}-y_{2}^{2}\right), \\
Q\left(\frac{y_{1}}{1-y_{3}}, \frac{y_{2}}{1-y_{3}}\right), \\
F_{3}\left(y_{1}, y_{2}, y_{3}\right)=y_{1}\left(1-y_{3}\right), P\left(\frac{y_{1}}{1-y_{3}}, \frac{y_{2}}{1-y_{3}}\right)+y_{2}\left(1-y_{3}\right), \\
Q\left(\frac{y_{1}}{1-y_{3}}, \frac{y_{2}}{1-y_{3}}\right) .
\end{gathered}
$$

This system is not defined at $p$, but it can be extended to $\mathbf{S}^{2}$ by introducing a change of time scale. We consider a new variable $u$ defined by $d t / d u=$ $\left(1-y_{3}\right)^{n}$. We then obtain the system $d y_{i} / d u=\left(1-y_{3}\right)^{n} F_{i}\left(y_{1}, y_{2}, y_{3}\right)$, for $i=1,2,3$, which extends analytically the flow of $\tilde{X}$ from $\mathbf{S}^{2} \backslash\{p\}$ to $\mathbf{S}^{2}$. We shall call the stereographic compactification of $X, s(X)$, the induced vector field in the two-sphere. Notice that the behaviour of the orbits of $X$ near infinity is determined by the behaviour of $s(X)$ near $p=(0,0,1)$. 
Remark 1.1. Let $Y$ be an analytic vector field on a two-dimensional manifold, and let $q$ be an isolated critical point of $Y$. Then we know that the local phase portrait of $Y$ at $q$ is either a focus, a center, or a finite union of elliptic, hyperbolic and parabolic sectors (for more details, see [L] or [ALGM]). From now on, we denote by $e=e(q)$ (resp. $h=h(q)$ ) the number of elliptic (resp. hyperbolic) sectors of $q$.

Proof of Proposition A. If $X$ is a bounded vector field, clearly there are no orbits of $s(X)$ whose $\omega$-limit is $p$ (otherwise, such an orbit would not be inside any compact set $\left.K \subset \mathbf{R}^{2}\right)$. So we have that $e(p)=h(p)=0$. Now, from Bendixson's index formula, $i=1+(e-h) / 2$ (see [ALGM]), we know that the index of $s(X)$ at $p$ is 1 .

Since all the critical points of $s(X)$ are isolated we can apply the PoincareHopf theorem, and assert that the sum of the indices at all the critical points of $s(X)$ is equal to 2, which is the Euler-Poincare characteristic of the two-sphere. Hence, the sum of the indices at all the critical points of $X$ is 1 .

\section{LOCAL STRUCTURE AT THE ISOLATED INFINITE CRITICAL POINTS FOR BOUNDED POLYNOMIAL VECTOR FIELDS IN THE PLANE}

From now onwards we shall use the Poincare compactification (see, for instance, [G, $\mathrm{S}$ or CGL]). Let $X$ be a polynomial vector field of degree $n$. First, we will consider that $\mathbf{R}^{2}$ is imbedded in $\mathbf{R}^{3}$ in such a way that, if $\left(y_{1}, y_{2}, y_{3}\right)$ represents an arbitrary point in $\mathbf{R}^{3}$, then $\mathbf{R}^{2}=\left\{\left(y_{1}, y_{2}, y_{3}\right) \in \mathbf{R}^{3}: y_{3}=1\right\}$. We then consider the sphere $\mathbf{S}^{2}=\left\{\left(y_{1}, y_{2}, y_{3}\right) \in \mathbf{R}^{3}: y_{1}^{2}+y_{2}^{2}+y_{3}^{2}=1\right\}$, the Poincaré sphere, which is tangent to $\mathbf{R}^{2}$ at the north pole $(0,0,1)$. After that, we consider the projection of the vector field $X$ in $\mathbf{R}^{2}$ onto $\mathbf{S}^{2}$ obtained by means of the central projections $p^{+}: \mathbf{R}^{2} \rightarrow \mathbf{S}^{2}$ and $p^{-}: \mathbf{R}^{2} \rightarrow \mathbf{S}^{2}$. That is, $p^{+}\left(y_{1}, y_{2}, 1\right)\left(\right.$ resp. $\left.p^{-}\left(y_{1}, y_{2}, 1\right)\right)$ is the intersection of the line joining $\left(y_{1}, y_{2}, 1\right)$ and the origin, with the northern (resp. southern) hemisphere in $\mathbf{S}^{2}$. We thus obtain an induced vector field in the northern and southern hemispheres. In each hemisphere the induced vector field is a copy of $X$.

We notice that the points at infinity of $\mathbf{R}^{2}$, two points for each direction, are now in a one-to-one correspondence with the points on the equator $\mathbf{S}^{1}=$ $\mathbf{S}^{2} \cap\left\{y_{3}=0\right\}$. We now attempt to extend the induced vector field on $\mathbf{S}^{2} \backslash \mathbf{S}^{1}$ to $\mathbf{S}^{2}$. However this vector field blows up as we approach $\mathbf{S}^{1}$ and the extension is not possible. Nonetheless, if we multiply it by the factor $y_{3}^{n-1}$ at each point $y \in \mathbf{S}^{2} \backslash \mathbf{S}^{1}$, the extension becomes possible. This extended field, which we call the Poincare compactification of $X$ and denote by $p(X)$, has $\mathbf{S}^{1}$ as an invariant set. The Poincare compactification for polynomial vector fields in $\mathbf{R}^{n}$ is given in Appendix 1.

In order to obtain the analytical expression for $p(X)$ we shall consider the two-sphere $\mathbf{S}^{2}$ as a differentiable manifold. We then choose six coordinate 
neighbourhoods given by $U_{i}=\mathbf{S}^{2} \cap\left\{y_{i}>0\right\}$ and $V_{i}=\mathbf{S}^{2} \cap\left\{y_{i}<0\right\}$, for $i=1,2,3$. The corresponding coordinate maps $F_{i}: U_{i} \rightarrow \mathbf{R}^{2}$ and $G_{i}: V_{i} \rightarrow$ $\mathbf{R}^{2}$ are defined by $F_{i}\left(y_{1}, y_{2}, y_{3}\right)=G_{i}\left(y_{1}, y_{2}, y_{3}\right)=\left(y_{j} / y_{i}, y_{k} / y_{i}\right)$ for $j<$ $k$ and $j, k \neq i$. We shall denote by $(y, z)$ the value of $F_{1}\left(y_{1}, y_{2}, y_{3}\right)=$ $G_{1}\left(y_{1}, y_{2}, y_{3}\right)$. The expressions for $p(X)$ in $V_{i}$ are those in $U_{i}$ multiplied by $(-1)^{n-1}$ for $i=1,2,3$. We note that the integral curves in $\mathbf{S}^{2}$ are always symmetric with respect to the origin of $\mathbf{R}^{3}$, but $p(X)$ is only symmetric when $n$ is odd. For instance, if $n$ is even and $\left(y_{1}, y_{2}, y_{3}\right) \in \mathbf{S}^{2}$ is a contracting node of $p(X)$ then $\left(-y_{1},-y_{2},-y_{3}\right)$ is an expanding node. We shall call finite (resp. infinite) critical points of $X$ or $p(X)$ the critical points of $p(X)$ which are in $\mathbf{S}^{2} \backslash \mathbf{S}^{1}$ (resp. $\mathbf{S}^{1}$ ).

Lemma 2.1. Let $X$ be a bounded polynomial vector field in the plane and let $q$ be an isolated infinite critical point. Then $q$ cannot be a focus or a centre. Furthermore, $e(q)=0$ and $h(q)$ is even.

Proof. We can assume, without loss of generality, that $q$ is in the local chart $U_{1}$. The critical point $q$ cannot be a focus or a centre because the straight line $z=0$ is invariant by the flow of $p(X)$. For the same reason, if an elliptic sector exists, its interior must be contained in $z>0$ or $z<0$, and the homoclinic orbits of this sector do not lie inside any compact set of $\mathbf{R}^{2}$; in that case the system will not be bounded. Then $e(q)=0$. From Bendixson's index formula (see $[\mathrm{ALGM}]$ ) we know that the number of elliptic and hyperbolic sectors have the same parity. Hence $e(q)=0$ implies that $h(q)$ is even.

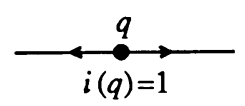

(a)

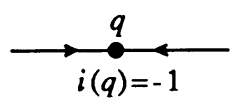

(b)

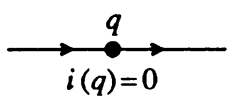

(c)

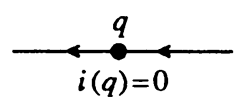

(d)

FIGURE 2.1. The index of one-dimensional vector field at an isolated critical point

Let $X: U \rightarrow \mathbf{R}^{k}$ be a vector field, where $U$ is an open set of $\mathbf{R}^{k}$. Let $q$ be an isolated critical point of $X$ in $U$. Then the function $X(u) /\|X(u)\|$ maps a small sphere, centered at $q$, into the unit $(k-1)$-sphere. It is well known that the degree of this mapping is called the index of $X$ at $q$ and is denoted by $i_{X}(q)$ or $i(q)$. If $k=1$ then the index at an isolated critical point can only be $0, \pm 1$. The local phase portrait at this critical point is described in Figure 2.1 (for more details, see $[\mathrm{M}]$ ).

Proposition 2.2. Let $X=(P, Q)$ be a bounded polynomial vector field of even degree in the plane. Suppose that $q$ is an isolated infinite critical point of $X$. Then the following hold:

(a) The local phase portrait of $p(X)$ at $q$ is described in Figure 2.2.

(b) $i_{p(X)}(q)=0$. 


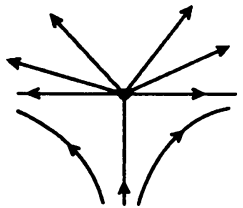

$\left(a_{1}\right)$

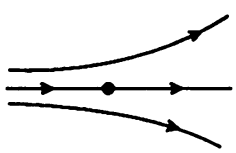

$\left(c_{1}\right)$

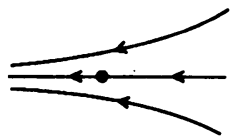

$\left(d_{1}\right)$

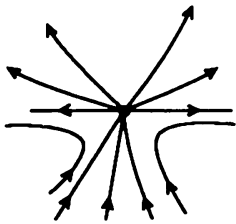

$\left(a_{2}\right)$

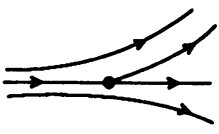

$\left(c_{2}\right)$

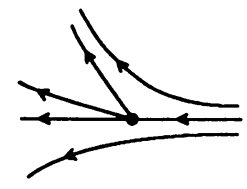

$\left(d_{2}\right)$

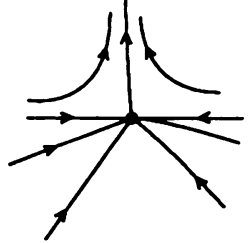

$\left(b_{1}\right)$
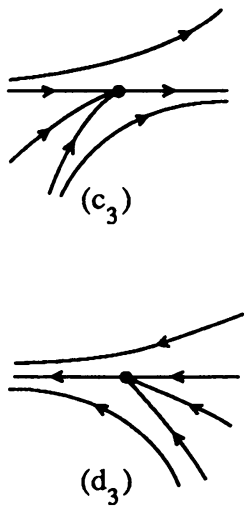

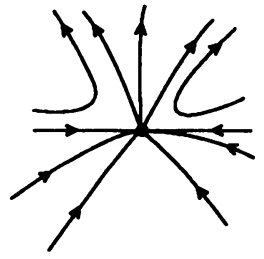

$\left(b_{2}\right)$

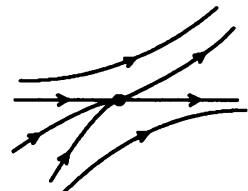

$\left(c_{4}\right)$

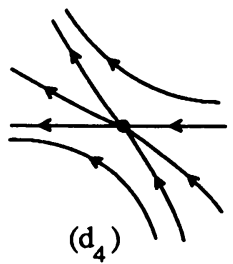

FIGURE 2.2. The local phase portrait of an isolated infinite critical point of a bounded polynomial vector field of even degree

Proof. We can assume that $q$ is in the local chart $U_{1}$ and that its coordinates $(y, z)$ are $(0,0)$. Given Bendixson's index formula and Lemma 2.1 all we need to show to prove (b) is that the number of hyperbolic sectors, $h(q)$, is two. Recall that the diametral opposite point of $q,(-q)$, is a critical point on $V_{1}$ and that $X$ is a bounded polynomial vector field of even degree. Then, there are no solutions in $\{z>0\} \cap U_{1}$ whose $\omega$-limit is $q$, and there are no solutions in $\{z<0\} \cap U_{1}$ whose $\alpha$-limit is $q$.

We have that, on $\{z=0\} \cap U_{1}, \dot{y}=F(y)=-y P_{n}(1, y)+Q_{n}(1, y)$ and $\dot{z}=0$, where $n$ is the degree of $X$ and $P_{n}$ and $Q_{n}$ are the homogeneous parts of degree $n$ of $P$ and $Q$, respectively. Since $(0,0)$ is an isolated critical point, $F(y)=a_{k} y^{k}+\cdots+a_{n+1} y^{n+1}$, where $k>0$ and $a_{k} \neq 0$. We consider three cases.

Case 1. $k$ is odd and $a_{k}>0$. In this case the local behaviour of the vector field at $q$ on $z=0$ is shown in Figure 2.1(a). By Remark 1.1 and Lemma 2.1 , if $h(q)=0, q$ will be a source and the system will not be bounded. Since $X$ is bounded it follows from Figure 2.3(a) that $q$ has no hyperbolic sectors in $z>0$, and that it has exactly two hyperbolic sectors in $z<0$. Hence the possible local phase portraits are drawn in Figures 2.2( $\left.\mathrm{a}_{1}\right)$ and 2.2( $\left.\mathrm{a}_{2}\right)$.

Case 2. $k$ is odd and $a_{k}<0$. By an argument similar to the one just used, it can be shown that $q$ must have two hyperbolic sectors in $z>0$ and no 


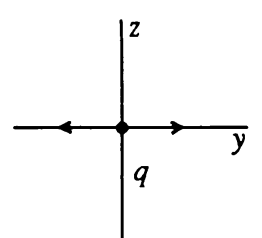

(a)

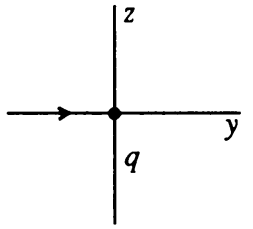

(b)

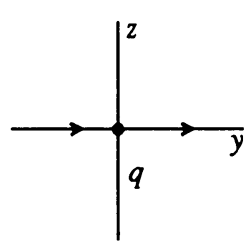

(c)

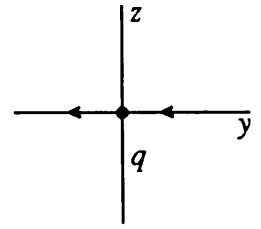

(d)

FIGURE 2.3. The behaviour of $p(X)$ on $z=0$

hyperbolic sectors in $z<0$. Therefore, the behaviour of $p(X)$ near $q$ is as drawn in Figures 2.2( $\left.b_{1}\right)$ and 2.2( $\left.b_{2}\right)$.

Case 3. $k$ is even. From Figures 2.3(c) and 2.3(d), Remark 1.1 and Lemma 2.1, it follows that $h(q)=2$ and $q$ has one hyperbolic sector in $z>0$ and another one in $z<0$. Since $q$ can have parabolic sectors, we obtain the remainder local phase portraits of Figure 2.2.

Remark 2.3. From the Poincaré-Hopf theorem on $\mathbf{S}^{2}$ (see [M]) we know that

$$
\sum_{\infty} i+2 \sum_{f} i=2
$$

where $\sum_{\infty} i$ denotes the sum of the indices of $p(X)$ at all the infinite critical points and $\sum_{f} i$ denotes the sum of the indices of $X$ at all the finite critical points. So, if the degree of $X$ is even, Proposition A can also be deduced from Proposition 2.2(b).

Proposition 2.4. Let $X=(P, Q)$ be a bounded polynomial vector field of odd degree in the plane. Suppose that $q$ is an isolated infinite critical point of $X$. Then the following hold:

(a) The local phase portrait of $p(X)$ at $q$ is described in Figure 2.4.

(b) $i_{p(X)}(q)=i_{p(X) \mid \mathbf{S}^{\prime}}(q)$, where $p(X) \mid \mathbf{S}^{1}$ denotes the restriction of the vector field $p(X)$ to the equator $\mathbf{S}^{1}$.

Proof. Assume that $q$ is in the local chart $U_{1}$ with coordinates $(y, z)=(0,0)$. Recall that $-q$ is also an infinite critical point and that the expression for $p(X)$ is the same in $U_{1}$ and in $V_{1}$ (because the degree $n$ of $X$ is odd). Since $X$ is bounded, there are no orbits in a neighbourhood of $q$ in $\mathbf{S}^{2} \backslash \mathbf{S}^{1}$ whose $\omega$-limit is $q$.

The expression for $p(X) \mid \mathbf{S}^{1}$ in $U_{1}$ is $(F(y), 0)$, where $F(y)=-y P_{n}(1, y)+$ $Q_{n}(1, y)$. Since $(0,0)$ is an isolated critical point, $F(y)=a_{k} y^{k}+\cdots+$ $a_{n+1} y^{n+1}$, with $k>0$ and $a_{k} \neq 0$. We consider three cases.

Case 1. $k$ is odd and $a_{k}>0$. From Figures 2.1(a) and 2.3(a), $i_{p(X) \mid \mathbf{S}^{\prime}}(q)=1$. Since $n$ is odd we deduce from Figure 2.3(a) that $h(q)=0$. From Lemma 2.1 and Bendixson's index formula, we obtain $i_{p(X)}(q)=1$. Furthermore, the only possible local phase portrait at $q$ is drawn in Figure 2.4(a). 
Case 2. $k$ is odd and $a_{k}<0$. From Figures 2.1(b) and 2.3(b), $i_{p(X) \mid \mathbf{S}^{1}}(q)=-1$. To see that $i_{p(X)}(q)=-1$ it is sufficient to show that $h(q)=4$. It follows from Lemma 2.1 that $h(q)$ is even. It is easy to see that if $h(q) \neq 4$ then $X$ is not bounded (see Figure 2.3(b)). Taking into account that $q$ can have parabolic sectors, the possible local phase portraits at $q$ are given in Figure 2.4 as cases $\left(b_{1}\right),\left(b_{2}\right),\left(b_{3}\right)$ and $\left(b_{4}\right)$.

Case 3. $k$ is even. From Figures 2.1(c), 2.1(d), 2.3(c) and 2.3(d), $i_{p(X) \mid \mathbf{S}^{1}}(q)$ $=0$. By Lemma 2.1, $e(q)=0$ and $h(q)$ is even. Then $h(q)>0$. If $h(q) \geq 4$ then $q$ has at least two stable separatrices and at least one of them is not contained in $z=0$. Hence $h(q)=2$. Therefore $i_{p(X)}(q)=0$. In this case, the local phase portraits at $q$, for $i \in\{1,2,3,4\}$, are drawn in Figures $2.4\left(\mathrm{c}_{i}\right)$ and $2.4\left(\mathrm{~d}_{i}\right)$.

Theorem B follows from Propositions 2.2 and 2.4.
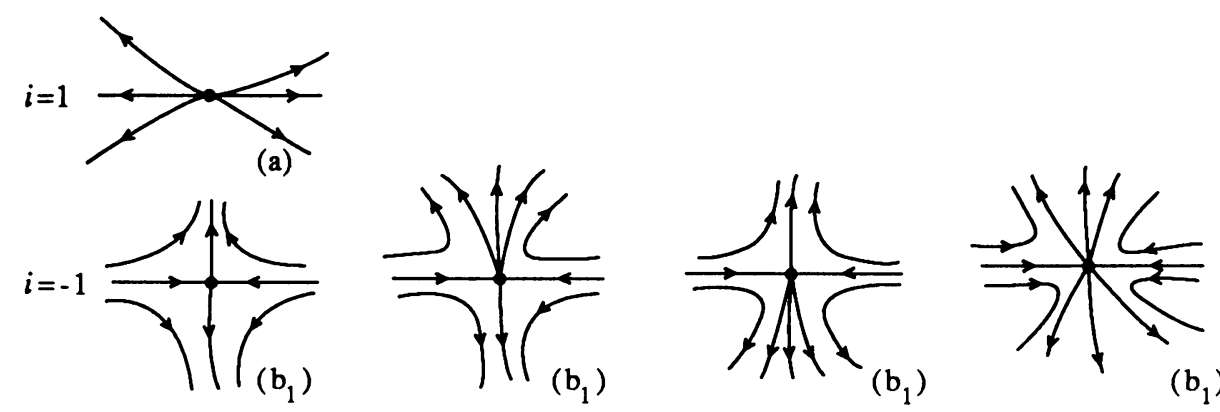

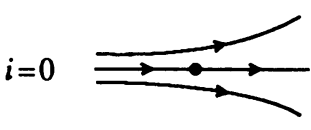

$\left(c_{1}\right)$
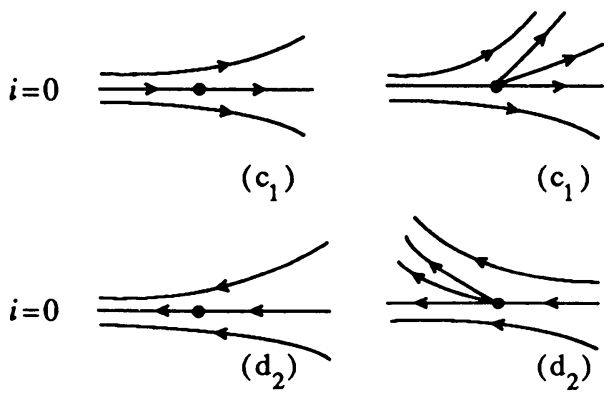

$\left(c_{1}\right)$
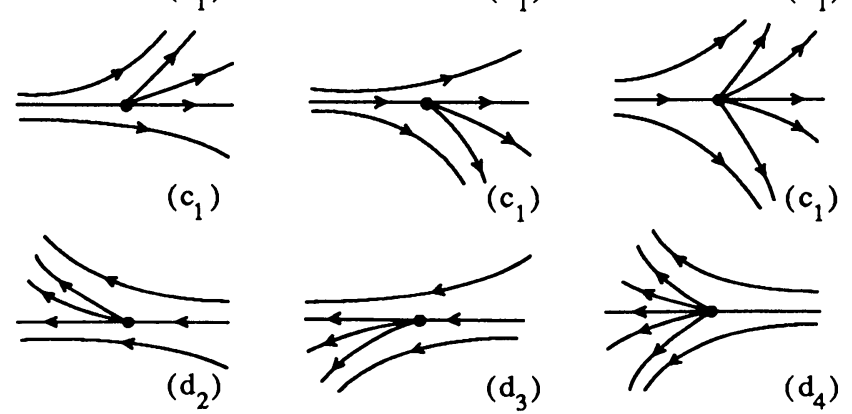

$\left(d_{4}\right)$

FIGURE 2.4. The local phase portraits of isolated infinite critical points of a bounded polynomial vector field of odd degree

Remark 2.5. Let $X$ be a polynomial vector field in $\mathbf{R}^{2}$ such that all its finite and infinite critical points are isolated. The Poincaré-Hopf theorem on $\mathbf{S}^{1}$ tells us that

$$
\sum i_{p(X) \mid \mathbf{S}^{1}}=\mathscr{G}\left(\mathbf{S}^{1}\right)=0,
$$

where the sum is over all the critical points of $p(X) \mid \mathbf{S}^{1}$ and $\mathscr{G}\left(\mathbf{S}^{1}\right)$ is the EulerPoincare characteristic of $\mathbf{S}^{1}$. So, if the degree of $X$ is odd, from Proposition 2.4(b) we obtain $\sum_{\infty} i=0$ and hence, as in Remark 2.3, Proposition A follows. 


\section{Classification of the phase-PORTRAits in A Neighbourhood of INFINITY OF BOUNDED CUBIC POLYNOMIAL SYSTEMS IN THE PLANE}

Let $q$ be a critical point of a vector field in the plane. We say that $q$ is elementary if there exists at least a nonzero eigenvalue of its linear part. We say that $q$ is degenerate if the determinant of its linear part is zero. The following result is well known (see for instance [ALGM]).

Theorem 3.1. Let $q$ be a critical point of an analytic vector field in the plane. If $q$ is nondegenerate then it is either a node, a focus, a centre or a saddle. If $q$ is degenerate and elementary then it is either a node, a saddle or a saddle-node with index $1,-1$ or 0 respectively.

Proposition 3.2. Let $\left(y_{j}, 0\right)$ be an infinite critical point on the local chart $U_{1}$ of a bounded polynomial vector field $X=(P, Q)$ of odd degree $n$. It follows that

(a) If $y_{j}$ is a root of odd (resp. even) multiplicity of $F(y)=-y P_{n}(1, y)+$ $Q_{n}(1, y)$, then the index at $\left(y_{j}, 0\right)$ is \pm 1 (resp. 0).

(b) If $y_{j}$ is a simple root of $F(y)$ then $\left(y_{j}, 0\right)$ is either a node or a saddle.

Proof. Since the expression for the vector field $X$ in the local chart $U_{1}$ is given by

$$
\left\{\begin{aligned}
\dot{y}= & {\left[-y P_{n}(1, y)+Q_{n}(1, y)\right]+z\left[-y P_{n-1}(1, y)+Q_{n-1}(1, y)\right] } \\
& +\cdots+z^{n}\left[-y P_{0}+Q_{0}\right] \\
\dot{z}= & -P_{n}(1, y) z-P_{n-1}(1, y) z^{2}-\cdots-P_{0} z^{n+1}
\end{aligned}\right.
$$

we have that $\dot{y}=F(y)$ on $z=0$. This, together with the proof of Proposition 2.4 , implies that the index at $\left(y_{j}, 0\right)$ must be \pm 1 when the multiplicity of $y_{j}$ is odd (Cases 1 and 2) and 0 when the multiplicity is even (Case 3). Hence (a) follows.

The linear part of the infinite critical point $\left(y_{j}, 0\right)$ is

$$
\left(\begin{array}{cc}
F^{\prime}\left(y_{j}\right) & * \\
0 & -P_{n}\left(1, y_{j}\right)
\end{array}\right)
$$

If $y_{j}$ is a simple root of $F(y)$ then $\left(y_{j}, 0\right)$ is elementary. From (a) and Theorem 3.1 we obtain (b).

Lemma 3.3. Let $X=(P, Q)$ be a cubic vector field and assume that $y=0$ is a double root of $F(y)$. Consider the system (3.1) and let $\theta(y, z)=y \dot{z}-z \dot{y}$. When $\dot{y}$ and $\dot{z}$ begin with terms of, at least, second order and $\theta_{3}$, the homogeneous part of third degree of $\theta$, is identically zero, then configurations $\left(c_{2}\right),\left(c_{3}\right),\left(d_{2}\right)$, and $\left(\mathrm{d}_{3}\right)$ in Figure 2.4 are not possible.

Proof. We can write $P_{3}(x, y)=r x^{3}+s x^{2} y+t x y^{2}+h y^{3}, Q_{3}(x, y)=d x^{3}+$ $e x^{2} y+f x y^{2}+g y^{3}, P_{2}(x, y)=a x^{2}+b x y+c y^{2}, Q_{2}(x, y)=\alpha x^{2}+\beta x y+\gamma y^{2}$, $P_{1}(x, y)=m x+n y, Q_{1}(x, y)=p x+q y, P_{0}=p_{0}$ and $Q_{0}=q_{0}$. Then we have that $F(y)=d+(e-r) y+(f-s) y^{2}+(g-t) y^{3}-h y^{4}$. Hence, $d=0$, 
$e-r=0$ and $f-s \neq 0$. System (3.1) can now be written as:

(3.2)

$$
\left\{\begin{aligned}
\dot{y}= & \alpha z+(f-s) y^{2}+(\beta-a) y z+p z^{2}+(g-t) y^{3}+(\gamma-b) y^{2} z \\
& +(q-m) y z^{2}+q_{0} z^{3}-h y^{4}-c y^{3} z-n y^{2} z^{2}-p_{0} y z^{3}, \\
\dot{z}= & -r z-s y z-a z^{2}-t y^{2} z-b y z^{2}-m z^{3}-h y^{3} z-c y^{2} z^{2}-n y z^{3}-p_{0} z^{4},
\end{aligned}\right.
$$

where $\alpha=r=0$. Since $\theta_{3}(y, z)=-z\left[f y^{2}+\beta y z+p z^{2}\right] \equiv 0$ then $f=\beta=$ $p=0$.

The directional blowing-ups $(y, z) \rightarrow(y, \lambda)$ defined by $z=\lambda y$, and $(y, z)$ $\rightarrow(w, z)$ defined by $y=w z$, allow us to write system (3.2) as the pair:

$$
\left\{\begin{aligned}
& \dot{y}=y^{2}\left[-s+(g-t) y-a \lambda-h y^{2}+(\gamma-b) y \lambda-c y^{2} \lambda\right. \\
&\left.+(q-m) y \lambda^{2}-n y^{2} \lambda^{2}+q_{0} y \lambda^{3}-p_{0} y^{2} \lambda^{3}\right], \\
& \dot{\lambda}=y^{2}\left[-\lambda\left(g+\gamma \lambda+q \lambda^{2}+q_{0} \lambda^{3}\right)\right]
\end{aligned}\right.
$$

and

$$
\left\{\begin{array}{l}
\dot{w}=z^{2}\left[q_{0}+q w+\gamma w^{2}+g w^{3}\right], \\
\dot{z}=z^{2}\left[-a-s w-m z-b w z-p_{0} z^{2}-t w^{2} z-n w z^{2}-c w^{2} z^{2}-h w^{3} z^{2}\right],
\end{array}\right.
$$

respectively. Call (3.5) and (3.6) the systems (3.3) and (3.4) after a change in time scale given by $d \tau=y^{2} d t$ and $d \tau=z^{2} d t$ respectively. Then system (3.5) on $y=0$ becomes $d y / d \tau=-s-a \lambda$ and $d \lambda / d \tau=-\lambda\left(g+\gamma \lambda+q \lambda^{2}+q_{0} \lambda^{3}\right)$, and system (3.6) on $z=0$ becomes $d w / d \tau=q_{0}+q w+\gamma w^{2}+g w^{3}$ and $d z / d \tau=-a-s w$.

When $q_{0}=0$ the straight line $y=0$ is invariant by the flow (3.2) and in consequence the local phase-portraits $\left(c_{2}\right),\left(c_{3}\right),\left(d_{2}\right)$ and $\left(d_{3}\right)$ in Figure 2.4 are not possible. So we shall assume that $q_{0} \neq 0$.

When $a=0$ we do not have any critical points on $y=0$ for system (3.5) (since $f-s=-s \neq 0$ ) or on $z=0$ for system (3.6) (since $q_{0} \neq 0$ ). Therefore $(y, z)=(0,0)$ has one hyperbolic sector in $z>0$, another one in $z<0$ and the number of parabolic and elliptic sectors is zero.

Assume now that $a \neq 0$. If there exists a critical point $(y, \lambda)$ for system (3.5) on $y=0$ which is different from $(0,0)$, then $(y, \lambda)=(0, \bar{\lambda})$, where $\bar{\lambda}=(-s / a) \neq 0$ is such that $q_{0} \bar{\lambda}^{3}+q \bar{\lambda}^{2}+\gamma \bar{\lambda}+g=0$. The linear part of $(0, \bar{\lambda})$ is

$$
\left(\begin{array}{cc}
(g-t)+(\gamma-b) \bar{\lambda}+(q-m) \bar{\lambda}^{2}+q_{0} \bar{\lambda}^{3} & -a \\
0 & -g-2 \gamma \bar{\lambda}-3 q \bar{\lambda}^{2}-4 q_{0} \bar{\lambda}^{3}
\end{array}\right) .
$$

From $\S 7, \S 8$ and Theorems 65 and 67 of [ALGM] and $a \neq 0$ we know all the local phase-portraits near $(y, \lambda)=(0, \bar{\lambda})$. Taking into account that $y=0$ is not invariant by the flow (3.5), we can draw all the local phase-portraits near $(y, z)=(0,0)$. We observe that configurations $\left(c_{2}\right),\left(c_{3}\right),\left(d_{2}\right)$ and $\left(d_{3}\right)$ in Figure 2.4. do not appear. 
Proposition 3.4. Let $X=(P, Q)$ be a cubic vector field and assume that $y=0$ is a double root of $F(y)$. If the local phase-portrait of $(y, z)=(0,0)$ for system (3.2) is topologically equivalent to $\left(\mathrm{c}_{2}\right),\left(\mathrm{c}_{3}\right),\left(\mathrm{d}_{2}\right)$ and $\left(\mathrm{d}_{3}\right)$ in Figure 2.4 , then $y=0$ is also a double root of $P_{3}(1, y)$.
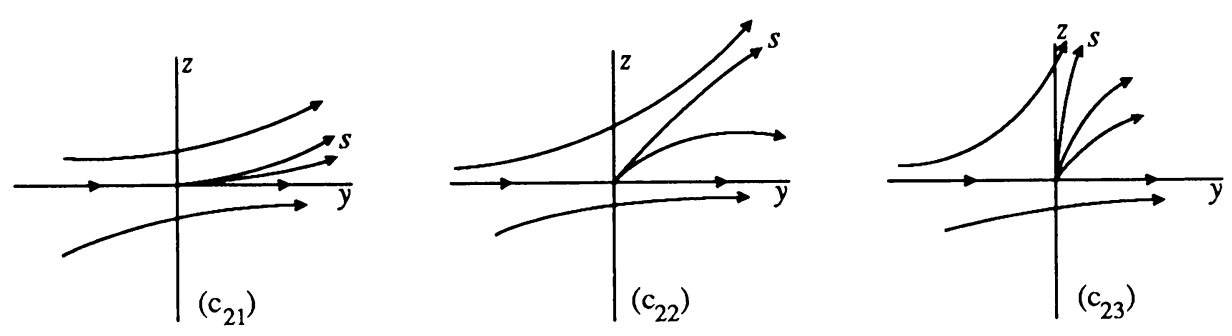

Figure 3.1. Saddle-nodes near $(y, z)=(0,0)$. The slope $\sigma$ of the separatrix $s$ at $(0,0)$ is $\sigma=0, \sigma \notin$ $\{0, \infty\}$ and $\sigma=\infty$ in cases $\left(\mathrm{c}_{21}\right),\left(\mathrm{c}_{22}\right)$ and $\left(\mathrm{c}_{23}\right)$, respectively

Proof. From (3.2) the linear part of $(y, z)=(0,0)$ is

$$
\left(\begin{array}{cc}
0 & \alpha \\
0 & -r
\end{array}\right)
$$

From Theorem 65 of $\left[\right.$ ALGM] and configurations $\left(c_{2}\right),\left(c_{3}\right),\left(d_{2}\right),\left(d_{3}\right)$ in Figure 2.4 , it follows that $r$ must be zero.

When $\alpha \neq 0$, the change of time scale given by $d \tau=\alpha d t$ allows us to apply Theorem 65 of [ALGM]. Hence, $\dot{y}=0$ implies $z=(-(f-s) / \alpha) y^{2}+$ higher-order terms and $\dot{z}\left(y,(-(f-s) / \alpha) y^{2}\right)=\left(s(f-s) / \alpha^{2}\right) y^{3}+$ higher-order terms. Since $(f-s) \neq 0, s=0$ is a necessary condition for the existence of a saddle node. In short, $P_{3}(1,0)=r=0$ and, since $\alpha \neq 0$, we have $\left.\left(d P_{3}(1, y) / d y\right)\right|_{y=0}=s=0$. Hence the proposition follows.

Assume $\alpha=0$. Consider the directional blowing-ups defined in Lemma 3.3, and get

$$
\left\{\begin{array}{c}
\dot{y}=y\left[(f-s) y+(g-t) y^{2}+(\beta-a) y \lambda-h y^{3}+(\gamma-b) y^{2} \lambda+p y \lambda^{2}\right. \\
\left.-c y^{3} \lambda+(q-m) y^{2} \lambda^{2}-n y^{3} \lambda^{2}+q_{0} y^{2} \lambda^{3}-p_{0} y^{3} \lambda^{3}\right], \\
\dot{\lambda}=y\left[-f \lambda-g y \lambda-\beta \lambda^{2}-\gamma y \lambda^{2}-p \lambda^{2}-q y \lambda^{3}-q_{0} y \lambda^{4}\right],
\end{array}\right.
$$

and

$$
\left\{\begin{array}{l}
\dot{w}=z\left[p+\beta w+q_{0} z+f w^{2}+q w z+\gamma w^{2} z+g w^{3} z\right] \\
\dot{z}=z\left[-a z-s w z-m z^{2}-b w z^{2}-p_{0} z^{3}-t w^{2} z^{2}-n w z^{3}-h w^{3} z^{3}\right]
\end{array}\right.
$$

Let $s$ be the only separatrix which is different from $z=0$. We consider three cases depending on the values of the slope of $s$ at the origin (see Figure 3.1). Similarly we could analyze the cases corresponding to the local phase portraits represented in $\left(d_{2}\right),\left(c_{3}\right)$ and $\left(d_{3}\right)$ in Figure 2.4. 
By Lemma 3.3, at least one of the coefficients $\beta, p$ or $f$, in systems (3.7) and (3.8), is different from zero. Also $f-s \neq 0$ because $y=0$ is a double root of $F(y)$.

Assume that we are in case $\left(c_{21}\right)$ in Figure 3.1. Since the slope of $s$ in $\left(c_{21}\right)$ is zero, it follows from (3.7) that either $p \neq 0$ and $\beta^{2}-4 p f<0$, or $p=\beta=0$ and $f \neq 0$. In short $f \neq 0$. This, together with (3.8), implies that $\beta^{2}-4 p f<0$. Therefore, $p f>0$. Since the linear part at $(y, \lambda)=(0,0)$ is

$$
\left(\begin{array}{cc}
f-s & 0 \\
0 & -f
\end{array}\right)
$$

the critical point $(y, \lambda)=(0,0)$ is a node or a saddle. If it is a node then $p f>0$ and in consequence the point $(y, z)=(0,0)$ is formed by two elliptic sectors. If it is a saddle then the point $(y, z)=(0,0)$ is formed by two hyperbolic sectors. Hence case $\left(c_{21}\right)$ is not possible.

Assume that we are in case $\left(c_{22}\right)$. Since the slope of $s$ in $\left(c_{22}\right)$ is not infinite, it follows from (3.8) that $p \neq 0$. Since the slope of $s$ in $\left(c_{22}\right)$ is different from zero we have, from (3.7), that $\beta^{2}-4 p f=0, \beta \neq 0$ and $f \neq 0$. Therefore, on the straight line $y=0$, the system $(3.7)$ has the critical points $(0,0)$ and $(0, \bar{\lambda})$ where $\bar{\lambda}=[-\beta /(2 p)] \neq 0$. The linear part at $(y, \lambda)=(0, \bar{\lambda})$ is

$$
\left(\begin{array}{cc}
f-s+(\beta-a) \bar{\lambda}+p \bar{\lambda}^{2} & 0 \\
-\bar{\lambda}\left(g+\gamma \bar{\lambda}+q \bar{\lambda}^{2}+q_{0} \bar{\lambda}^{3}\right) & 0
\end{array}\right) .
$$

If $(f-s)+(\beta-a) \bar{\lambda}+p \bar{\lambda}^{2}=-s-a \bar{\lambda} \neq 0$ then (by Theorem 65 of [ALGM]), $(y, \lambda)=(0, \bar{\lambda})$ is a node, a saddle or a saddle-node. Using its linear part it follows that $(y, \lambda)=(0,0)$ is a node or a saddle. When we draw the local phase-portrait at $(y, z)=(0,0)$ we see that $\left(c_{22}\right)$ is not possible. Therefore, assume that $s+a \bar{\lambda}=0$. If $g+\gamma \bar{\lambda}+q \bar{\lambda}^{2}+q_{0} \bar{\lambda}^{3}=0$, then on the straight line $\lambda=\bar{\lambda}$ we have that $\dot{\lambda}=-\bar{\lambda}\left(f+\beta \bar{\lambda}+p \bar{\lambda}^{2}\right)-\bar{\lambda} y\left(g+\gamma \bar{\lambda}+q \bar{\lambda}^{2}+q_{0} \bar{\lambda}^{3}\right)=0$. So the straight line $z=\bar{\lambda} y$ is invariant by the flow of (3.2). Again, case $\left(c_{22}\right)$ is not possible. Hence, $g+\gamma \bar{\lambda}+q \bar{\lambda}^{2}+q_{0} \bar{\lambda}^{3} \neq 0$. With the coordinates $(Y, \Lambda)$ defined by $Y=y$ and $\Lambda=\lambda-\bar{\lambda}$, the system (3.7) becomes

$$
\left\{\begin{array}{l}
\dot{Y}=C_{1} Y^{2}-a Y \Lambda+\text { higher-order terms, } \\
\dot{\Lambda}=D_{1} Y+D_{2} Y \Lambda+\frac{1}{2} \beta \Lambda^{2}+\text { higher-order terms, }
\end{array}\right.
$$

where

$$
\begin{aligned}
& C_{1}=g-t+(\gamma-b) \bar{\lambda}+(q-m) \bar{\lambda}^{2}+q_{0} \bar{\lambda}^{3}, \\
& D_{1}=-g \bar{\lambda}-\gamma \bar{\lambda}-q \bar{\lambda}^{3}-q_{0} \bar{\lambda}^{4} \neq 0, \\
& D_{2}=-g-2 \gamma \bar{\lambda}-3 q \bar{\lambda}^{2}-4 q_{0} \bar{\lambda}^{3} .
\end{aligned}
$$

We now change the time scale by $d s=D_{1} d t$ so as to be able to apply Theorem 67 in [ALGM]. Let $Y=\left(-\beta / 2 D_{1}\right) \Lambda^{2}+\cdots$ be the solution of $\dot{\Lambda}=0$ in a neighbourhood of $(0,0)$. Then, $\dot{Y}\left(\left(-\beta / 2 D_{1}\right) \Lambda^{2}+\cdots, \Lambda\right)=\left(\alpha \beta / 2 D_{1}^{2}\right) \Lambda^{3}+\cdots$. 
In order to obtain $\left(c_{22}\right)$ we apply Theorem 67 and deduce that $(y, \lambda)=(0, \bar{\lambda})$ must be a saddle-node. Hence $\dot{Y}\left(\left(-\beta / 2 D_{1}\right) \Lambda^{2}+\cdots, \Lambda\right)$ must have a first term of even order, i.e., $a=0$. From $s+a \bar{\lambda}=0$ it follows that $\left.\left(d P_{3}(1, y) / d y\right)\right|_{y=0}=$ $s=0$. Therefore, since $P_{3}(1,0)=r=0$, we have that $y=0$ is a double root of $P_{3}(1, y)$.

Assume that we are in case $\left(c_{23}\right)$. We need the point $(0,0)$ to be the only critical point of system (3.8); in particular $p=0$. Also $(0,0)$ must be the unique critical point of system (3.7) on $y=0$, i.e., $\beta=0$ or $f=0$. But $f=0$ implies that the linear part at $(y, \lambda)=(0,0)$ is

$$
\left(\begin{array}{cc}
-s & 0 \\
0 & 0
\end{array}\right)
$$

where $s \neq 0$ since $f-s \neq 0$. Then, by Theorem 65 in [ALGM], $(y, \lambda)=(0,0)$ is a saddle-node with the parabolic sector filling the region $\lambda>0$ or $\lambda<0$ depending on the sign of $\beta$. Therefore there are orbits of the system (3.2) outside $z=0$ such that their $\alpha$-limit is the critical point $(y, z)=(0,0)$. Hence, since we are in case $\left(c_{23}\right), f \neq 0$ and $\beta=p=0$. So the linear parts of systems (3.7) and (3.8) at the critical points $(y, \lambda)=(0,0)$ and $(w, z)=(0,0)$ are, respectively,

$$
\left(\begin{array}{cc}
f-s & 0 \\
0 & -f
\end{array}\right) \text { and }\left(\begin{array}{cc}
0 & q_{0} \\
0 & -a
\end{array}\right)
$$

Therefore, $(y, \lambda)=(0,0)$ is a node or a saddle. Since $(y, z)=(0,0)$ is of type $\left(\mathrm{c}_{23}\right)$, it must be a saddle. Notice that $q_{0} \neq 0$; otherwise the straight line $y=0$ would be invariant by the flow of (3.2) in contradiction with the local behaviour of $\left(\mathrm{c}_{23}\right)$. When $a \neq 0$, by Theorem 65 of [ALGM], there are orbits of system (3.2) in $z<0$ and $z>0$ such that their $\alpha$-limit or $\omega$-limit is the critical point $(y, z)=(0,0)$. These orbits come from orbits of system (3.8) which start or end at $(w, z)=(0,0)$ in the direction $q_{0} z+a w=0$. So $a=0$. We can now apply Theorem 67 of [ALGM] after changing the time scale by $d s=q_{0} d t$. Since $(y, z)=(0,0)$ is a saddle-node of type $\left(c_{23}\right),(w, z)=(0,0)$ in system (3.8) must be a saddle-node. Let $z=\left(-f / q_{0}\right) w^{2}+\cdots$ be the solution of $\dot{w}=0$ in a neighbourhood of the origin. Then $\dot{z}=\left(s f / q_{0}^{2}\right) w^{3}+$ higher-order terms. To obtain a saddle-node at $(w, z)=(0,0), \dot{z}$ must have a first term of even order in $w$; so $s=0$. Also, in this case, $P_{3}(1,0)=\left.\left(d P_{3}(1, y) / d y\right)\right|_{y=0}=0$. The proposition is now proved.

Proof of Theorem C. Let $X=(P, Q)$ be a bounded cubic system in the plane and set $F(x, y)=x Q(x, y)-y P(x, y)$. Assume, for instance, that $F$ has one double real root $y_{1}$ and two simple real roots $y_{2}, y_{3}$. By Proposition 3.2, the critical points $\left(y_{2}, 0\right)$ and $\left(y_{3}, 0\right)$ are a node or a saddle, and $\left(y_{1}, 0\right)$ has zero index. By Proposition 2.4, we can have three possibilities for $\left(y_{1}, 0\right)$ determining configurations (2), (3) or (4) in Figure 1.1.

When $F$ has two double real roots we know, from Propositions 3.2 and 2.4, that there exist six possible kinds of behaviour at infinity. However, for cubic 
vector fields, only five of them are possible. They are (5), (6), (7), (8) and (9) in Figure 1.1. By Proposition 3.4, two saddle-nodes at infinity of type $\left(c_{2}\right),\left(c_{3}\right)$, $\left(d_{2}\right)$ or $\left(d_{3}\right)$ cannot coexist. In this way we obtain Figure 1.1.

Table 1.1 follows in a straightforward way by using $\S 7, \S 8$, Theorem 65 and 67 of $[\mathrm{ALGM}]$ and the blow-up techniques.

The method applied to classify the phase-portraits for the bounded cubic systems with isolated infinite critical points, in a neighbourhood of infinity, also works for any bounded polynomial system with isolated infinite critical points. The classification for the bounded linear (resp. quadratic) systems is easy (resp. known, see [DP]). No specific work has been done for bounded systems of degree greater than three.

\section{GENERIC INDEX FOR BOUNDED POLYNOMIAL VECTOR FIELDS IN $\mathbf{R}^{n}$}

Let

$$
X=\left(P^{1}, P^{2}, \ldots, P^{n}\right)
$$

be a polynomial vector field in $\mathbf{R}^{n}$ with $\operatorname{degree}\left(P^{i}\right)=m$ for $i=1,2, \ldots, n$. In this section we shall give a generalization from $\mathbf{R}^{2}$ to $\mathbf{R}^{n}$ of Proposition $A$. It is well known that if $q \in \mathbf{R}^{n}$ is an isolated critical point of $X$, the index of $X$ at $q$ can be computed as the sum of the signs of the jacobian of $X$ at all the $X$-preimages near $q$ of a regular value of $X$ near 0 (see [M]).

The expression for the Poincaré compactification of $X, p(X)$, in the local chart $U_{1}$ is given by

$$
\left\{\begin{array}{l}
\dot{z}_{1}=\left[-z_{1} P_{m}^{1}+P_{m}^{2}\right]+z_{n}\left[-z_{1} P_{m-1}^{1}+P_{m-1}^{2}\right]+\cdots+z_{n}^{m}\left[-z_{1} P_{0}^{1}+P_{0}^{2}\right], \\
\dot{z}_{2}=\left[-z_{2} P_{m}^{1}+P_{m}^{3}\right]+z_{n}\left[-z_{2} P_{m-1}^{1}+P_{m-1}^{3}\right]+\cdots+z_{n}^{m}\left[-z_{1} P_{0}^{1}+P_{0}^{3}\right], \\
\vdots \\
\dot{z}_{n}=-z_{n} P_{m}^{1}-z_{n}^{2} P_{m-1}^{1}-\cdots-z_{n}^{m+1} P_{0}^{1},
\end{array}\right.
$$

where $z_{1}, z_{2}, \ldots, z_{n}$ are the local coordinates of $U_{1}$ and

$$
P_{k}^{i} \equiv P_{k}^{i}\left(1, z_{1}, \ldots, z_{n-1}\right) .
$$

The critical points at infinity in the local chart $U_{1}$ are points $\left(z_{1}, z_{2}, \ldots, z_{n}\right)$ where $z_{n}=0$ and $z_{1}, z_{2}, \ldots, z_{n-1}$ are given by the solutions of the system

$$
\left\{\begin{array}{l}
-z_{1} P_{m}^{1}\left(1, z_{1}, \ldots, z_{n-1}\right)+P_{m}^{2}\left(1, z_{1}, \ldots, z_{n-1}\right)=0, \\
-z_{2} P_{m}^{1}\left(1, z_{1}, \ldots, z_{n-1}\right)+P_{m}^{3}\left(1, z_{1}, \ldots, z_{n-1}\right)=0, \\
\vdots \\
-z_{n-1} P_{m}^{1}\left(1, z_{1}, \ldots, z_{n-1}\right)+P_{m}^{n}\left(1, z_{1}, \ldots, z_{n-1}\right)=0 .
\end{array}\right.
$$

Assume that $O=(0, \ldots, 0)$ is a critical point of (4.1). Denote by $Z=$ 
$\left(\dot{z}_{1}, \dot{z}_{2}, \ldots, \dot{z}_{n}\right)$. From (4.1) we obtain

$$
(D Z)(0)=\left(\begin{array}{cccc}
\left(\frac{\partial \dot{z}_{1}}{\partial z_{1}}\right)(0) & \cdots & \left(\frac{\partial \dot{z}_{1}}{\partial z_{n-1}}\right)(0) & \left(\frac{\partial \dot{z}_{1}}{\partial z_{n}}\right)(0) \\
\vdots & & \vdots & \vdots \\
\left(\frac{\partial \dot{z}_{n_{1}}}{\partial z_{1}}\right)(0) & \cdots & \left(\frac{\partial \dot{z}_{n-1}}{\partial z_{n-1}}\right)(0) & \left(\frac{\partial \dot{z}_{n-1}}{\partial z_{n}}\right)(0) \\
0 & \cdots & 0 & \left(\frac{\partial \dot{z}_{n}}{\partial z_{n}}\right)(0)
\end{array}\right) .
$$

Hence $\operatorname{det}(D Z)(0)=\left(\partial \dot{z}_{n} / \partial z_{n}\right)(0) \operatorname{det}\left(D Z^{\prime}\right)(0)$ where $Z^{\prime}$ is the restriction of $Z$ to $z_{n}=0$.

Lemma 4.1. Assume that $\left(z_{1}, z_{2}, \ldots, z_{n}\right)=(0, \ldots, 0)=O$ is a critical point of system (4.1) and that $\left(\partial \dot{z}_{n} / \partial z_{n}\right)(0)>0$ (resp. $\left.\left(\partial \dot{z}_{n} / \partial z_{n}\right)(0)<0\right)$. Then there exist some orbits $\gamma_{1}(t)$ and $\gamma_{2}(t)$ contained in $\left\{z_{n}>0\right\}$ and $\left\{z_{n}<0\right\}$ respectively, such that their $\alpha$-limit (resp. $\omega$-limit) is 0 .

Proof. Call $a=\left(\partial \dot{z}_{n} / \partial z_{n}\right)(0)$ and $A=(D Z)(0)$. It follows from (4.3) that $\operatorname{det}(A-a I)=0$; i.e., $a$ is an eigenvalue of $A$. Assume $a>0$ (resp. $a<0$ ) and divide the spectrum of $A$ into three parts $\sigma_{s}, \sigma_{c}, \sigma_{u}$ such that

$$
\operatorname{Re} \lambda \begin{cases}<0 & \text { if } \lambda \in \sigma_{s}, \\ =0 & \text { if } \lambda \in \sigma_{c}, \\ >0 & \text { if } \lambda \in \sigma_{u} .\end{cases}
$$

Let the generalized eigenspaces associated to $\sigma_{s}, \sigma_{c}$ and $\sigma_{u}$ be $E^{s}, E^{c}$ and $E^{u}$ respectively. By the Center Manifold Theorem there exist stable and unstable invariant manifolds $W^{s}$ and $W^{u}$ which are tangent to $E^{u}$ and $E^{s}$ at 0 and a center manifold $W^{c}$ which is tangent to $E^{c}$ at 0 .

Since $a \in \sigma_{u}$ (resp. $\left.a \in \sigma_{s}\right), \operatorname{Ker}(A-a I)^{\alpha} \subset E^{u}$ (resp. $\operatorname{Ker}(A-a I)^{\alpha} \subset$ $E^{s}$ ), where $\alpha$ is the multiplicity of the root $a$ of the characteristic polynomial $\operatorname{det}(A-\lambda I)=0$. So, $\operatorname{dim} \operatorname{Ker}(A-a I)^{\alpha}=\alpha$ and $\operatorname{dim} \operatorname{Ker}(A-a I)^{\beta}=\alpha$ for each $\beta \geq \alpha$ (see [HS]). We claim that $\operatorname{Ker}(A-a I)^{\alpha} \not \subset\left\{z_{n}=0\right\}$. To see this let $v^{1}, v^{2}, \ldots, v^{\alpha}$ be a basis of $\operatorname{Ker}(A-a I)^{\alpha}$ and assume that $v^{i}=$ $\left(z_{1}^{i}, z_{2}^{i}, \ldots, z_{n}^{i}\right)$ satisfies $z_{n}^{i}=0$ for each $i=1,2, \ldots, \alpha$. Writing

$$
A=\left(\begin{array}{cccc} 
& & & a_{1} \\
& B & & \vdots \\
& & & a_{n-1} \\
0 & \cdots & 0 & a
\end{array}\right)
$$

a simple computation gives

$$
(A-a I)^{\alpha}=\left(\begin{array}{cccc} 
& & & c_{1} \\
& (B-a I)^{\alpha} & & \vdots \\
& & & c_{n-1} \\
0 & \cdots & 0 & 0
\end{array}\right) .
$$


For each $i=1,2, \ldots, \alpha$, the equation $(A-a I)^{\alpha}\left(v^{i}\right)=0$ implies that

$$
(B-a I)^{\alpha} w^{i}=0, \quad \text { where } w^{i}=\left(z_{1}^{i}, \ldots, z_{n-1}^{i}\right) .
$$

Since the vectors $w^{i}$ are linearly independent, $\alpha \leq \operatorname{dim} \operatorname{Ker}(B-a I)^{\alpha}$. Since $\operatorname{det}(A-a I)=(a-\lambda) \operatorname{det}(B-\lambda I)$, when $a$ is a root of $\operatorname{det}(A-\lambda I)=0$ of multiplicity $\alpha, a$ is a root of $\operatorname{det}(B-\lambda I)=0$ of multiplicity $(\alpha-1)$. So, $\operatorname{dim} \operatorname{Ker}(B-a I)^{\alpha-1}=\alpha-1=\operatorname{dim} \operatorname{Ker}(B-a I)^{\alpha}$, which is a contradiction. The claim is proved.

Since $\operatorname{Ker}(A-a I)^{\alpha} \subset E^{u}$ (resp. $\left.\operatorname{Ker}(A-a I)^{\alpha} \subset E^{s}\right), E^{u} \not \subset\left\{z_{n}=0\right\}$ (resp. $\left.E^{s} \not \subset\left\{z_{n}=0\right\}\right)$. Hence $E^{u}$ (resp. $E^{s}$ ) intersects transversally the hyperplane $\left\{z_{n}=0\right\}$. Since $W^{u}$ (resp. $\left.W^{s}\right)$ is tangent to $E^{u}$ (resp. $E^{s}$ ), the lemma follows.

Proposition 4.2. Let $X=\left(P^{1}, P^{2}, \ldots, P^{n}\right) \in \mathscr{G}_{m}$ be a bounded polynomial vector field in $\mathbf{R}^{n}$ such that $X$ has some infinite critical points and $\operatorname{degree}\left(P^{i}\right)=$ $m$, for all $i=1,2, \ldots, n$. Then the following hold:

(a) $m$ is odd.

(b) If $\left(z_{1}, \ldots, z_{n}\right)=(0, \ldots, 0)$ is a critical point of system (4.1) then $a=$ $\left(\partial \dot{z}_{n} / \partial z_{n}\right)(0)>0$.

Proof. Let $q$ be an infinite critical point. Without loss of generality we can assume that $q$ is in the local chart $U_{1}$ and its coordinates are $\left(z_{1}, \ldots, z_{n}\right)=$ $(0, \ldots, 0)$. This implies that $P_{m}^{i}(1,0, \ldots, 0)=0$ for each $i=2,3, \ldots, n$ (see (4.2)). Since $X \in \mathscr{G}_{m}, a=-P_{m}^{1}(1,0, \ldots, 0)$ must be different from zero. Otherwise, system $P_{m}^{i}(1,0, \ldots, 0)=0$, for $i=1,2, \ldots, n$, has the root $(1,0, \ldots, 0)$.

Assume that $m$ is even. Then, by the Poincare compactification, if $q$ is an infinite critical point in the local chart $U_{1},-q$ is an infinite critical point in the local chart $V_{1}$. Furthermore, the orbits near $q$ in $z_{n}>0$ are the orbits near $-q$ in $z_{n}<0$ with reversed orientation. By Lemma 4.1, since $a \neq 0$, there exists some orbit of $X$ whose $\omega$-limit is $q$ or $-q$. So, the system will not be bounded. Therefore (a) is proved.

Assume now that $m$ is odd. Then $a$ satisfies $a>0$. Otherwise, by Lemma 4.1 , there exists some orbit whose $\omega$-limit is $q$ and $X$ will not be bounded. Hence, (b) follows.

Proof of Theorem D. Since the equator of $\mathbf{S}^{n}$ is invariant by the flow of $p(X)$, each infinite critical point of $X$ determines a critical point of $\left.p(X)\right|_{\mathbf{S}^{n-1}}$. Applying the Poincaré-Hopf theorem on $\mathbf{S}^{n-1}$ we get

$$
\left.\sum i_{p(X) \mid}\right|_{\mathrm{s}^{n-1}}=\chi\left(\mathbf{S}^{n-1}\right)=1+(-1)^{n-1}= \begin{cases}2 & \text { if } n \text { is odd }, \\ 0 & \text { if } n \text { is even. }\end{cases}
$$

We assume first that $X$ has no infinite critical points. Then $n$ must be even. By the Poincaré-Hopf theorem on $\mathbf{S}^{n}$

$$
\sum i_{p(X)}=\chi\left(\mathbf{S}^{n}\right)=1+(-1)^{n}=2 .
$$


On the other hand,

$$
\sum i_{p(X)}=\sum_{U_{n+1}} i_{p(X)}+\sum_{V_{n+1}} i_{p(X)}+\sum_{\mathbf{S}^{n-1}} i_{p(X)}=2 \sum_{f} i_{X}+\sum_{\infty} i_{X} .
$$

Since $\sum_{\infty} i_{X}=0$ we obtain $\sum_{f} i_{X}=1=(-1)^{n}$.

Now we assume that $X$ has infinite critical points. Let $q$ be one of them. We claim that the index of $p(X)$ at $q$ is the same as the index of $\left.p(X)\right|_{\mathbf{S}^{n-1}}$ at $q$. To see this, assume that $q$ is in the local chart $U_{1}$ and its coordinates are $\left(z_{1}, \ldots, z_{n}\right)=(0, \ldots, 0)$. By Proposition $4.2, a=-P_{m}^{1}(1,0, \ldots, 0)>$ 0 . Let $G_{m}\left(z_{1}, \ldots, z_{n}\right)$ be such that $\dot{z}_{n}=z_{n} G_{m}\left(z_{1}, \ldots, z_{n}\right)$ (see system (4.1)). Since $G_{m}(0, \ldots, 0)=-P_{m}^{1}(1,0, \ldots, 0)>0$, the algebraic hypersurface $G_{m}\left(z_{1}, \ldots, z_{n}\right)=0$ does not contain $(0, \ldots, 0)$. So, let $\delta$ be such that:

(i) $G_{m}\left(z_{1}, \ldots, z_{n}\right)>0$ for all $z \in B_{\delta}(0)=\left\{z \in \mathbf{R}^{n}:\|z\|<\delta\right\}$.

(ii) Inside $B_{\delta}(0)$ there are no critical points of $Z$ different from 0 .

Call $\mathbf{S}_{\delta}^{n-1}=\partial B_{\delta}(0)$ and consider the map $\mathbf{S}_{\delta}^{n-1} \rightarrow \mathbf{S}^{n-1}$ defined by $z \rightarrow$ $Z(z) /\|Z(z)\|$. To compute the degree of this map we select a regular value of $Z$ that has all its preimages in the hyperplane $z_{n}=0$. We can take, for instance, $z_{\delta}=(\delta, 0, \ldots, 0)$, because if $Z(z)=Z\left(z_{\delta}\right)$ then $\dot{z}_{n}(z)=0$ and by (i) it implies that $z_{n}=0$ (if $z_{\delta}$ is not regular, we can vary $\delta$ slightly). Let $\left\{z^{1}, \ldots, z^{k}\right\}$ be the set of all preimages of $Z\left(z_{\delta}\right)$ in $\mathbf{S}_{\delta}^{n-1}$. Writing $z^{i}=\left(z_{1}^{i}, \ldots, z_{n-1}^{i}, z_{n}^{i}\right)$ and denoting by $y^{i}=\left(z_{1}^{i}, \ldots, z_{n-1}^{i}\right)$ and $y_{\delta}=$ $(\delta, 0, \ldots, 0) \in \mathbf{R}^{n-1}$, it follows that the set $\left\{y^{1}, y^{2}, \ldots, y^{k}\right\}$ is composed of the preimages of $y_{\delta}$ by $Z^{\prime}$, where $Z^{\prime}$ is the restriction of $Z$ to $z_{n}=0$. From (4.1) we obtain $(D Z)\left(z^{i}\right)=\left(D Z^{\prime}\right)\left(y^{i}\right) G_{m}\left(z^{i}\right)$. Since $G_{m}>0$ in $B_{\delta}(0)$, the claim follows.

Applying the Poincaré-Hopf theorem on $\mathbf{S}^{n-1}$ and $\mathbf{S}^{n}$ we obtain, respectively,

$$
\sum i_{p(X) / \mathbf{S}^{n-1}}=\chi\left(\mathbf{S}^{n-1}\right)=\sum_{\infty} i_{X}
$$

and

$$
\sum i_{p(X)}=2 \sum_{f} i_{X}+\sum_{\infty} i_{X}=\chi\left(\mathbf{S}^{n}\right) .
$$

Since $\chi\left(\mathbf{S}^{n}\right)-\chi\left(\mathbf{S}^{n-1}\right)=2(-1)^{n}$ we deduce

$$
\sum_{f} i_{X}=(-1)^{n}
$$

\section{Appendix 1. The Poincaré compactification}

Let $X=\left(P^{1}, P^{2}, \ldots, P^{n}\right)$ be a polynomial vector field in $\mathbf{R}^{n}$ and let $m=$ $\max \left\{\operatorname{deg}\left(P^{1}\right), \operatorname{deg}\left(P^{2}\right), \ldots, \operatorname{deg}\left(P^{n}\right)\right\}$ be the degree of $X$. 
Consider the hyperplane $\Pi=\left\{x \in \mathbf{R}^{n+1}: x_{n+1}=1\right\}$ in $\mathbf{R}^{n+1}$ and let $\mathbf{S}^{n}=$ $\left\{y \in \mathbf{R}^{n+1}:\|y\|=1\right\}$ be the $n$-sphere in $\mathbf{R}^{n+1}$. Since the origin of $\mathbf{R}^{n+1}$ is the center of $\mathbf{S}^{n}$, for each $p=\left(x_{1}, \ldots, x_{n}, 1\right) \in \Pi$ the vector $\frac{1}{\Delta(x)}\left(x_{1}, \ldots, x_{n}, 1\right)$, where $\Delta(x)=\left(1+\sum_{i=1}^{n} x_{i}^{2}\right)^{1 / 2}$ belongs to $H_{+}=\left\{y \in \mathbf{S}^{n}: y_{n+1}>0\right\}$ while the vector $\frac{-1}{\Delta(x)}\left(x_{1}, \ldots, x_{n}, 1\right)$ belongs to $H_{-}=\left\{y \in \mathbf{S}^{n}: y_{n+1}<0\right\}$. So we can define the epimorphisms

$$
f_{+}: \mathbf{R}^{n} \rightarrow H_{+} \subset \mathbf{S}^{n} \text { and } f_{-}: \mathbf{R}^{n} \rightarrow H_{-} \subset \mathbf{S}^{n},
$$

by $f_{+}(x)=\frac{1}{\Delta(x)}\left(x_{1}, \ldots, x_{n}, 1\right)$ and $f_{-}(x)=\frac{-1}{\Delta(x)}\left(x_{1}, \ldots, x_{n}, 1\right)$. In this manner $X$ induces a vector field $\tilde{X}$ in $H_{+} \cup H_{-}$defined by $\tilde{X}(y)=\left(D f_{+}\right)_{x} X(x)$ when $y=f_{+}(x)$, and by $\widetilde{X}(y)=\left(D f_{-}\right)_{x} X(x)$ when $y=f_{-}(x)$.

The expression for $\tilde{X}(y)$ on $H_{+} \cup H_{-}$is

$$
\widetilde{X}(y)=y^{n+1}\left(\begin{array}{ccccc}
1-y_{1}^{2} & -y_{2} y_{1} & -y_{3} y_{1} & \cdots & -y_{n} y_{1} \\
-y_{1} y_{2} & 1-y_{2}^{2} & -y_{3} y_{2} & \cdots & -y_{n} y_{2} \\
\vdots & \vdots & \vdots & & \vdots \\
-y_{1} y_{n} & -y_{2} y_{n} & -y_{3} y_{n} & \cdots & 1-y_{n}^{2} \\
-y_{1} y_{n+1} & -y_{2} y_{n+1} & -y_{3} y_{n+1} & \cdots & -y_{n} y_{n+1}
\end{array}\right)\left(\begin{array}{c}
P^{1} \\
P^{2} \\
\vdots \\
P^{n}
\end{array}\right) \text {, }
$$

where $P^{i} \equiv P^{i}\left(y_{1} / y_{n+1}, \ldots, y_{n} / y_{n+1}\right)$ or $P^{i} \equiv P^{i}\left(-y_{1} / y_{n+1}, \ldots,-y_{n} / y_{n+1}\right)$ depending on whether $y_{n+1}>0$ or $y_{n+1}<0$, respectively. This defines an analytical vector field defined on the whole of $\mathbf{S}^{n}$, namely,

$$
y_{n+1}^{m-1} \tilde{X}(y) .
$$

This vector field is called the Poincaré compactification of $X$ and it is denoted by $p(X)$.

To obtain the analytical expression for $p(X)$ we shall consider the $n$-sphere as a differentiable manifold. We choose the $2 n+2$ coordinate neighbourhoods given by $U_{i}=\left\{y \in \mathbf{S}^{n}: y_{i}>0\right\}$ and $V_{i}=\left\{y \in \mathbf{S}^{n}: y_{i}<0\right\}$, for $i=1,2, \ldots, n+1$. The corresponding coordinate maps $F_{i}: U_{i} \rightarrow \mathbf{R}^{n}$ and $G_{i}: V_{i} \rightarrow \mathbf{R}^{n}$ are defined by

$$
F_{i}(y)=G_{i}(y)=\frac{1}{y_{i}}\left(y_{j_{1}}, y_{j_{2}}, \ldots, y_{j_{n}}\right)
$$

with $1 \leq j_{1}<j_{2}<\cdots<j_{n} \leq n+1$, and $j_{k} \neq i$, for all $k=1,2, \ldots, n$. We now do the computations on $U_{1}$. Let $y \in U_{1} \cap H_{+}$. Then, $\left(D F_{1}\right)_{y}: T_{y} U_{1} \rightarrow$ $T_{F_{1}(y)} \mathbf{R}^{n}$ and

$$
\left(D F_{1}\right)_{y}\left(y_{n+1}^{m-1} \tilde{X}(y)\right)=y_{n+1}^{m-1}\left(D F_{1}\right)_{y}\left(D f_{+}\right)_{x} X(x)=y_{n+1}^{m-1} D\left(F_{1} \circ f_{+}\right)_{x} X(x),
$$

where $y=f_{+}(x)$. Then,

$$
D\left(F_{1} \circ f_{+}\right)_{x} X(x)=\frac{1}{x_{1}^{2}}\left(-x_{2} P^{1}+x_{1} P^{2},-x_{3} P^{1}+x_{1} P^{3}, \ldots,-x_{n} P^{1}+x_{1} P^{n},-P^{1}\right) \text {, }
$$

where $P^{i} \equiv P^{i}\left(x_{1}, x_{2}, \ldots, x_{n}\right)$. 
Let $\left(z_{1}, z_{2}, \ldots, z_{n}\right)$ be the coordinates on $U_{1}$, that is:

$$
\left(z_{1}, z_{2}, \ldots, z_{n}\right)=F_{1}\left(y_{1}, y_{2}, \ldots, y_{n+1}\right)=\left(\frac{y_{2}}{y_{1}}, \frac{y_{3}}{y_{1}}, \ldots, \frac{y_{n+1}}{y_{1}}\right) \text {. }
$$

Then (2) becomes

(3)

$D\left(F_{1} \circ f_{+}\right)_{x} X(x)=z_{n}\left(-z_{1} P^{1}+P^{2},-z_{2} P^{1}+P^{3}, \ldots,-z_{n-1} P^{1}+P^{n},-z_{n} P^{1}\right)$,

where $P^{i} \equiv P^{i}\left(1 / z_{n}, z_{1} / z_{n}, \ldots, z_{n-1} / z_{n}\right)$.

Since $y_{n+1}^{m-1}=\left[z_{n} / \Delta(z)\right]^{m-1}$, the vector field (1) becomes

$$
\frac{z_{n}^{m}}{\Delta(z)^{m-1}}\left(-z_{1} P^{1}+P^{2},-z_{2} P^{1}+P^{3}, \ldots,-z_{n-1} P^{1}+P^{n},-z_{n} P^{1}\right) .
$$

If $y \in U_{1} \cap H_{-}$we get the same expression we obtained in (4). In a similar manner we can deduce the expressions for $p(X)$ in $U_{2}, \ldots, U_{n}$. These are, respectively:

$$
\begin{gathered}
\frac{z_{n}^{m}}{\Delta(z)^{m-1}}\left(-z_{1} P^{2}+P^{1},-z_{2} P^{2}+P^{3}, \ldots,-z_{n-1} P^{2}+P^{n},-z_{n} P^{2}\right), \\
\text { where } P^{i} \equiv P^{i}\left(\frac{z_{1}}{z_{n}}, \frac{1}{z_{n}}, \ldots, \frac{z_{n-1}}{z_{n}}\right) ; \\
\vdots \\
\frac{z_{n}^{m}}{\Delta(z)^{m-1}}\left(-z_{1} P^{n}+P^{1},-z_{2} P^{n}+P^{2}, \ldots,-z_{n-1} P^{n}+P^{n-1},-z_{n} P^{n}\right), \\
\text { where } P^{i} \equiv P^{i}\left(\frac{z_{1}}{z_{n}}, \ldots, \frac{z_{n-1}}{z_{n}}, \frac{1}{z_{n}}\right) .
\end{gathered}
$$

The expression for $p(X)$ in $U_{n+1}$ is $z_{n}^{m+1}\left(P^{1}, P^{2}, \ldots, P^{n}\right)$, where $P^{i} \equiv$ $P^{i}\left(z_{1}, z_{2}, \ldots, z_{n}\right)$.

On the other hand, the expression for $p(X)$ in the local chart $V_{i}$ is the same as in $U_{i}$ multiplied by $(-1)^{m-1}$. Notice that in $V_{i}, y_{n+1}^{m-1}=\left[-z_{n} / \Delta(z)\right]^{m-1}=$ $(-1)^{m-1}\left[z_{n} / \Delta(z)\right]^{m-1}$.

The vector field $p(X)$ allows us to study the behaviour of $X$ in the neighbourhood of infinity, that is, in the neighbourhood of the equator $\mathbf{S}^{n-1}=$ $\left\{y \in \mathbf{S}^{n}: y_{n+1}=0\right\}$. From the expressions for $p(X)$ in the local charts $U_{i}$ and $V_{i}$, for $i=1,2, \ldots, n+1$, we deduce that the infinity is invariant by the flow of $p(X)$ and that $p(X)$ has two copies of $X$ on the northern and southern hemisphere of $\mathbf{S}^{n}$, that is, on $U_{n+1}$ and $V_{n+1}$, respectively.

We shall call finite (resp. infinite) critical points of $X$ or of $p(X)$, the critical points of $p(X)$ which lie on $\mathbf{S}^{n} \backslash \mathbf{S}^{n-1}$ (resp. $\mathbf{S}^{n-1}$ ).

Notice that the integral curves in $\mathbf{S}^{n}$ are always symmetric with respect to the origin of $\mathbf{R}^{n+1}$, but the vector field is only symmetric when $m$ is odd. 
Furthermore, due to this symmetry, if $y \in \mathbf{S}^{n-1}$ is an infinite critical point then $-y$ is another one.

\section{APPENDIX 2. GENERICITY}

Let $\mathscr{X}_{m}$ be the set of polynomial vector fields $X=\left(P^{1}, P^{2}, \ldots, P^{n}\right)$ defined on $\mathbf{R}^{n}$ with degree $\left(P^{i}\right)=m_{i}$ and $m=\left(m_{1}, m_{2}, \ldots, m_{n}\right)$. We can identify $\mathscr{X}_{m}$ with $\mathbf{R}^{N}$, where $N$ is the number of coefficients of all the $P^{i}$, for $i=1,2, \ldots, n$. Let $\mathscr{G}_{m}$ be the set of all $X \in \mathscr{X}_{m}$ such that all the solutions of system $P^{i}=0$, for $i=1,2, \ldots, n$, lie on the "finite part" of $\mathbf{R}^{n}$. That is, if $\bar{P}^{i}$ is the homogeneous polynomial in the variables $x_{0}, x_{1}, \ldots, x_{n}$ of degree $m_{i}$ such that $\bar{P}^{i}\left(1, x_{1}, \ldots, x_{n}\right)=P^{i}\left(x_{1}, x_{2}, \ldots, x_{n}\right)$ and degree $\left(P^{i}\right)=$ degree $\left(\bar{P}^{i}\right)$, then the solutions of system $\bar{P}^{i}=0$, for $i=1,2, \ldots, n$, with $x_{0}=0$, are in the hyperplane at infinity. So, $X \in \mathscr{G}_{m}$ means that system $\bar{P}^{i}=0$, for $i=1,2, \ldots, n$, with $x_{0}=0$ has only the trivial solution $x_{0}=x_{1}=\cdots=x_{n}=0$. Notice that this system is equivalent to system $P_{m_{i}}^{i}=0$, for $i=1,2, \ldots, n$, where $P_{m_{i}}^{i}$ is the homogeneous part of $P^{i}$ of maximum degree.

We want to see that $X \in \mathscr{G}_{m}$ is a "generic" condition. For this we need the concept of the resultant of two polynomials (see [S]).

Let $A(x, y)=a_{0} x^{m}+a_{1} x^{m-1} y+\cdots+a_{m} y^{m}$ and $B(x, y)=b_{0} x^{n}+b_{1} x^{n-1} y+$ $\cdots+b_{n} y^{n}$ be two homogeneous polynomials associated to the points $\left(a_{0}, a_{1}\right.$, $\left.\ldots, a_{m}\right) \in \mathbf{R}^{m+1}$ and $\left(b_{0}, b_{1}, \ldots, b_{n}\right) \in \mathbf{R}^{n+1}$. We claim that there exists a polynomial $R$ of degree $(m+1)(n+1)$ in the variables $a_{0}, a_{1}, \ldots, a_{m}, b_{0}, b_{1}$, $\ldots, b_{n}$, with the following property: if $A$ and $B$ have some common linear factor, then $R(A, B)=0$. This polynomial is known as the resultant of polynomials $A$ and $B$.

Assume $A=C \bar{A}$ and $B=C \bar{B}$, and let $(\bar{x}, \bar{y}) \neq(0,0)$ be such that $C(\bar{x}, \bar{y})=0$. Multiplying $A(x, y)$ successively by $x^{n-1}, x^{n-2} y, \ldots, y^{n-1}$ and $B(x, y)$ by $x^{m-1}, x^{m-2} y, \ldots, y^{m-1}$ and renaming $x^{m+n-1}, x^{m+n-2} y$, $\ldots, y^{m+n-1}$ by $z_{1}, x_{2}, \ldots, z_{m+n}$ we obtain

$$
\begin{aligned}
& a_{0} z_{1}+a_{1} z_{2}+\cdots+a_{m} z_{m+1}=0, \\
& a_{0} z_{2}+a_{1} z_{3}+\cdots+a_{m} z_{m+2}=0, \\
& \vdots \\
& a_{0} z_{n}+a_{1} z_{n+1}+\cdots+a_{n} z_{m+n}=0, \\
& b_{0} z_{1}+b_{1} z_{2}+\cdots+b_{n} z_{n+1}=0, \\
& b_{0} z_{2}+b_{1} z_{3}+\cdots+b_{n} z_{n+2}=0, \\
& \vdots \\
& b_{0} z_{m}+b_{1} z_{m+1}+\cdots+b_{n} z_{n+m}=0 .
\end{aligned}
$$


This is a homogeneous linear system of $n+m$ equations with a nontrivial solution $\left(\bar{z}_{1}, \bar{z}_{2}, \ldots, \bar{z}_{n+m}\right)=\left(\bar{x}^{m+n-1}, \bar{x}^{m+n-2} \bar{y}, \ldots, \bar{y}^{m+n-1}\right)$. Hence, its determinant must be zero. We define $R(A, B)$ to be the determinant of the above system. $R(A, B)$ is a polynomial in the coefficients of $A$ and $B$ and it satisfies the claim.

Proposition 1. $\mathscr{X}_{m} \backslash \mathscr{G}_{m}$ is contained in an algebraic hypersurface of $\mathscr{X}_{m}$; i.e. there exists a polynomial function $\Phi: \mathscr{X}_{m} \rightarrow \mathbf{R}$, such that $\mathscr{X}_{m} \backslash \mathscr{G}_{m} \subset \Phi^{-1}(0)$. Proof. Let $X=\left(P^{1}, P^{2}, \ldots, P^{n}\right) \in \mathscr{X}_{m}$ where $m=\left(m_{1}, m_{2}, \ldots, m_{n}\right)$. Consider the system:

$$
P_{m_{i}}^{i}\left(1, x_{2}, \ldots, x_{n}\right)=0, \quad \text { for } i=1, \ldots, n .
$$

We define:

$$
\begin{aligned}
& R_{j}=R\left(P_{m_{1}}^{1}\left(1, x_{2}, \ldots, x_{n}\right), P_{m_{j}}^{j}\left(1, x_{2}, \ldots, x_{n}\right)\right), \quad \text { for } j-2, \ldots, n, \\
& \text { when interpreting } P_{m_{1}}^{1} \text { and } P_{m_{j}}^{j} \text { as polynomials in } x_{2} \text {; } \\
& R_{2 j}=R\left(R_{2}, R_{j}\right), \quad \text { for } j=3, \ldots, n \text {, } \\
& \text { when interpreting } R_{2} \text { and } R_{j} \text { as polynomials in } x_{3} \text {; } \\
& R_{23 j}=R\left(R_{23}, R_{2 j}\right), \quad \text { for } j=4, \ldots, n \text {, } \\
& \text { when interpreting } R_{23} \text { and } R_{2 j} \text { as polynomials in } x_{4} \text {; } \\
& R_{23 \cdots(n-2) j}=R\left(R_{23 \cdots(n-3)(n-2)}, R_{23 \cdots(n-3) j}\right), \quad \text { for } j=n-1, n \text {; } \\
& \text { when interpreting } R_{23 \cdots(n-3)(n-2)} \text { and } R_{23 \cdots(n-3) j} \text { as polynomials in } x_{n-1} \text {; } \\
& R_{23 \cdots n}=R\left(R_{23 \cdots(n-2)(n-1)}, R_{23 \cdots(n-2) n}\right) \\
& \text { when interpreting } R_{23 \cdots(n-2)(n-1)} \text { and } R_{23 \cdots(n-2) n} \text { as polynomials in } x_{n} \text {. }
\end{aligned}
$$

Notice that $R_{23 \cdots n}$ depends only on the coefficients of $P_{m_{i}}^{i}$, for $i=1, \ldots, n$. We claim that if one of the roots of the system $P_{m_{i}}^{i}\left(x_{1}, x_{2}, \ldots, x_{n}\right)=0$, for $i=1,2, \ldots, n$, is such that $x_{1} \neq 0$, then $R_{23 \cdots n}$ must be zero. To see this, let $\bar{y}_{j}$ be defined by $\bar{y}_{j}=x_{j} / x_{1}$, for $j=1,2, \ldots, n$, where $\left(x_{1}, x_{2}, \ldots, x_{n}\right)$ is a solution of the system $P_{m_{i}}^{i}\left(x_{1}, x_{2}, \ldots, x_{n}\right)=0$. From the homogeneity of $P_{m_{1}}^{i}$ we obtain that $P_{m_{i}}^{i}\left(1, \bar{y}_{2}, \ldots, \bar{y}_{n}\right)=0$, for $i=1,2, \ldots, n$. So, the polynomials $P_{m_{1}}^{i}\left(1, x_{2}, \bar{y}_{3}, \ldots, \bar{y}_{n}\right)$ have the root $x_{2}=\bar{y}_{2}$ in common. Then

$$
R_{j}\left(\bar{y}_{3}, \ldots, \bar{y}_{n}\right)=0, \quad \text { for } j=2, \ldots, n \text {. }
$$

Consider now the system $R_{j}\left(x_{3}, \bar{y}_{4}, \ldots, \bar{y}_{n}\right)=0$. Since the polynomials $R_{j}$ have the root $x_{3}=\bar{y}_{3}$ in common, we have that $R_{2 j}=R\left(R_{2}, R_{j}\right)=0$. In a similar way we obtain that $R_{23 \cdots n}$ must be zero. We shall call $\Phi_{1}$ the polynomial $R_{23 \cdots n}$. 
Following the same procedure but beginning with systems

$$
P_{m_{j}}^{j}\left(x_{1}, \ldots, x_{i-1}, 1, x_{i+1}, \ldots, x_{n}\right)=0, \quad \text { for } j=1,2, \ldots, n ;
$$

we can construct the polynomials $\Phi_{1}, \Phi_{2}, \ldots, \Phi_{n}$. Now set $\Phi=\Phi_{1} \Phi_{2} \ldots \Phi_{n}$. We claim that $\mathscr{X}_{m} \backslash \mathscr{G}_{m} \subset \Phi^{-1}(0)$. Let $X \in \mathscr{X}_{m} \backslash \mathscr{G}_{m}$; i.e. assume that there exists a solution $\left(x_{1}, x_{2}, \ldots, x_{n}\right)$ of system $P_{m_{i}}^{i}\left(x_{1}, x_{2}, \ldots, x_{n}\right)=0$, for $i=$ $1,2, \ldots, n$, where $x_{j} \neq 0$ for some $j \in\{1,2, \ldots, n\}$. Then, $\Phi_{j}(X)=0$ and consequently $\Phi(X)=0$.

\section{REFERENCES}

[ALGM] A. A. Andronov, E. A. Leontovich, I. I. Gordon and A. L. Maier, Qualitative theory of second order dynamic systems, Wiley, 1973.

[C] A. Cima, Indices of polynomial vector fields with applications, Ph.D. Thesis, Universitat Autònoma de Barcelona, 1987.

[CGL] B. Coll, A. Gasull and J. Llibre, Some theorems on the existence, uniqueness and nonexistence of limit cycles for quadratic systems, J. Differential Equations 67 (1987), 372-399.

[DP] R. J. Dickson and L. M. Perko, Bounded quadratic systems in the plane, J. Differential Equations 7 (1970), 251-273.

[G] E. A. V. Gonzales, Generic properties of polynomial vector fields at infinity, Trans. Amer. Math. Soc. 143 (1969), 201-222.

[GH] J. Guckenheimer and P. Holmes, Nonlinear oscillations, dynamical systems and bifurcation of vector fields, Springer-Verlag, 1983.

[HS] M. Hirsch and S. Smale, Differential equations, dynamical systems and linear algebra, Academic Press, 1974.

[L] S. Lefschetz, Differential equations: Geometric theory, Interscience, 1962.

[M] J. Milnor, Topology from a differentiable viewpoint, University Press of Virginia, Charlottesville, Virginia, 1965.

[S] J. Sotomayor, Curvas definidas por equacoes diferenciais no plano, Inst. Mat. Pura e Aplicada, Rio de Janeiro, 1981.

Departament de Matemàtiques, Facultat de Ciències, Universitat Autònoma de Barcelona, 08193 Bellaterra, Barcelona, Spain 